\title{
Thermodynamics of Superfluidity
}

\author{
A.F. Andreev and L.A. Melnikovsky
}

\begin{abstract}
New, superfluid specific additive integral of motion is found. This facilitates investigation of general thermodynamic equilibrium conditions for superfluid. The analysis is performed in an extended space of thermodynamic variables containing (along with the usual thermodynamic coordinates such as pressure and temperature) superfluid velocity and momentum density. The equilibrium stability conditions lead to thermodynamic inequalities which replace the Landau superfluidity criterion at finite temperatures.

KEY WORDS: superfluidity, thermodynamics, critical velocity
\end{abstract}

\section{INTRODUCTION}

Existence of two independent macroscopic motions is a specific feature of superfluids. Equilibrium state is, thus, characterized by two velocities of these motions. In general the equilibrium thermodynamic state is unambiguously determined by the values of all additive integrals of motion. Integral of motion that corresponds to one of the velocities (the "normal component velocity"), like it is in usual hydrodynamics, is momentum.

Appearance of the superfluid velocity as a thermodynamic variable implies the existence of an extra additive integral of motion. Below we explicitly find the integral and this allows us to investigate general thermodynamic stability conditions and, in particular, the problem of critical velocities.

Usually in experiments the vortices destroy superfluidity at Feynman critical velocity which is far below the Landau critical velocity. This is why the superfluid hydrodynamics equations can be expanded in powers of low velocities and one safely uses the first nontrivial terms of this expansion.

Nevertheless, there is a number of experiments 1, 2] where this is not enough. In some of them the superfluid flow is studied in small orifices. It has been shown that in these circumstances the maximum velocity is inversely proportional to the orifice width and may reach the order of the Landau critical velocity if the aperture is small enough. Other experiments are performed at temperatures extremely close to the superfluid transition. In this region, the linear approximation fails at low velocities starting from those much smaller than the Feynman critical velocity (which does not depend on the temperature). This means that all thermodynamic quantities of the superfluid become nontrivial functions of the not small superfluid velocity (i.e., they depend not only on the 
usual thermodynamic coordinates such as pressure and temperature). The only assumption one can make (and we do it) is that the fluid at rest is isotropic. This quite general statement of the problem is used in the paper (and in our earlier letter [3]); in the next section we find the complete set of thermodynamic inequalities in this light, i.e., the conditions imposed on thermodynamic functions for the superfluid to remain stable.

Later we employ the Landau phonon-roton model to calculate the highest velocity compatible with obtained thermodynamic inequalities and show that it can be interpreted as a critical velocity. This thermodynamic scenario supposedly explains the superfluidity breakdown in small orifices. In the vicinity of the $\lambda$-point we use scaling speculations to find critical behavior of the critical velocity.

\section{STABILITY}

Thermodynamic equilibrium of an isolated system is a state with maximum entropy at given values of all its additive integrals of motion.

Superfluid system is unusual in the sense it has a continuous gauge symmetry spontaneously broken, such system possesses a specific quantum order parameter, "wave function of the BE condensate". This is why in superfluid there are two independent velocities of normal and superfluid motion. The superfluid velocity is (up to a factor) a gradient of the wave function phase $\mathbf{v}_{\mathrm{s}}=\nabla \phi$. The same is true for the time derivative $\dot{\mathbf{v}}_{\mathrm{s}}=\nabla \dot{\phi}$. This is a local conservation law of all three components of the vector $\mathbf{V}_{\mathrm{s}}=\int \mathbf{v}_{\mathrm{s}} \mathrm{d} \mathbf{r}$. In other words, $\mathbf{v}_{\mathrm{s}}$ is the density of a new additive integral of motion (along with mass density or momentum density). This integral is specific to superfluid.

Instead of the entropy maximality, it is usually convenient 4 to use another requirement, equivalent to the first one, namely the energy minimality under constant entropy and additive integrals of motion. This is the requirement of thermodynamic stability and may be expressed in form of thermodynamic inequalities.

Total energy of the superfluid $E_{\text {tot }}$ is an integral of the energy density $E$ over the entire volume $E_{\mathrm{tot}}=\int E \mathrm{~d} \mathbf{r}$. The energy density can be obtained via a Galilean transformation [ $[$ ]

$$
E=\frac{\rho v_{\mathrm{s}}^{2}}{2}+\mathbf{v}_{\mathrm{s}} \mathbf{j}_{0}+E_{0} .
$$

Here, $\mathbf{v}_{\mathbf{s}}$ is the superfluid velocity, $\rho$ is the mass density and subscript "0" denotes quantities measured in the frame of reference of the superfluid component (that is the frame, where the superfluid velocity is zero). Namely, $E_{0}$ and $\mathbf{j}_{0}$ are the energy density and the momentum density with respect to the superfluid component. The former is a function of $\rho, \mathbf{j}_{0}$, and the entropy density $S$. Its differential can be written as

$$
\mathrm{d} E_{0}=T \mathrm{~d} S+\mu \mathrm{d} \rho+\mathbf{w} \mathrm{d} \mathbf{j}_{0},
$$


where Lagrange multipliers $T, \mu$, and $\mathbf{w}$ are the temperature, the chemical potential, and the so-called relative velocity of normal and superfluid components.

The liquid is isotropic and, consequently, the velocity $\mathbf{w}$ and the momentum density $\mathbf{j}_{0}$ are parallel to each other:

$$
\mathbf{j}_{0}=j_{0}(T, \rho, w) \frac{\mathbf{w}}{w} .
$$

This leads to a useful identity for the partial derivatives of $\mathbf{j}_{0}$ with respect to w:

$$
\left(\frac{\partial j_{0}^{k}}{\partial w^{l}}\right)_{T, \rho}=\frac{w^{k} w^{l}}{w^{2}}\left(\frac{\partial j_{0}}{\partial w}\right)_{T, \rho}+\left(\frac{\delta^{k l}}{w}-\frac{w^{k} w^{l}}{w^{3}}\right) j_{0} .
$$

Further transforming (1), we can write $\mathrm{d} E$ with the help of (2) in the form

$$
\mathrm{d} E=T \mathrm{~d} S+\left(\mu+\frac{v_{\mathrm{s}}^{2}}{2}-\mathbf{v}_{\mathrm{s}} \mathbf{v}_{\mathrm{n}}\right) \mathrm{d} \rho+\left(\mathbf{j}-\rho \mathbf{v}_{\mathrm{n}}\right) \mathrm{d} \mathbf{v}_{\mathrm{s}}+\mathbf{v}_{\mathrm{n}} \mathrm{d} \mathbf{j},
$$

where $\mathbf{j}=\rho \mathbf{v}_{\mathrm{s}}+\mathbf{j}_{0}$ is the total momentum density and $\mathbf{v}_{\mathrm{n}}=\mathbf{v}_{\mathrm{s}}+\mathbf{w}$ is the normal velocity.

Stability implies that each "allowed" fluctuation increases the total energy of the system $E_{\text {tot }}$. Allowed are the fluctuations leaving conserved quantities unchanged. This means that the minimality of $E_{\text {tot }}$ must be investigated under fixed entropy and all additive integrals of motion: mass, momentum, and superfluid velocity.

Consider a macroscopic fluctuation of all the variables $\delta S, \delta \rho, \delta \mathbf{v}_{\mathrm{s}}$, and $\delta \mathbf{j}$. They are conserved and this ensures that the first variation of the total energy for a uniform system is identically zero

$$
\begin{aligned}
\delta E_{\mathrm{tot}}=\int\left\{\left(\frac{\partial E}{\partial S}\right)_{\rho, \mathbf{v}_{\mathrm{s}}, \mathbf{j}} \delta S+\left(\frac{\partial E}{\partial \rho}\right)_{S, \mathbf{v}_{\mathrm{s}}, \mathbf{j}} \delta \rho+\right. & \left.\left(\frac{\partial E}{\partial \mathbf{v}_{\mathrm{s}}}\right)_{S, \rho, \mathbf{j}} \delta \mathbf{v}_{\mathrm{s}}+\left(\frac{\partial E}{\partial \mathbf{j}}\right)_{S, \rho, \mathbf{v}_{\mathrm{s}}} \delta \mathbf{j}\right\} \mathrm{d} \mathbf{r} \equiv 0 .
\end{aligned}
$$

The matrix of the second variation quadratic form is a Jacobian matrix $8 \times 8$

$$
\mathfrak{H}=\left\|\frac{\partial\left(T, \mathbf{v}_{\mathbf{n}}, \mu+v_{\mathrm{s}}^{2} / 2-\mathbf{v}_{\mathbf{s}} \mathbf{v}_{\mathbf{n}}, \mathbf{j}-\rho \mathbf{v}_{\mathbf{n}}\right)}{\partial\left(S, \mathbf{j}, \quad \mathbf{v}_{\mathbf{s}}\right)}\right\| .
$$

It is positive definite if all principal minors in the top-left corner $M_{1}, M_{2}, \ldots M_{8}$ are positive. We recursively test these minors:

- The first positivity condition

$$
\begin{aligned}
& M_{1}=\frac{\partial\left(T, \mathbf{j}, \rho, \mathbf{v}_{\mathrm{s}}\right)}{\partial\left(S, \mathbf{j}, \rho, \mathbf{v}_{\mathrm{s}}\right)}= \frac{\partial\left(T, \mathbf{j}, \rho, \mathbf{v}_{\mathrm{s}}\right)}{\partial\left(T, \mathbf{v}_{\mathrm{n}}, \rho, \mathbf{v}_{\mathrm{s}}\right)} \frac{\partial\left(T, \mathbf{v}_{\mathrm{n}}, \rho, \mathbf{v}_{\mathrm{s}}\right)}{\partial\left(S, \mathbf{j}, \rho, \mathbf{v}_{\mathrm{s}}\right)}= \\
&\left(\frac{\partial j_{0}}{\partial w}\right)_{T, \rho}\left(\left(\frac{\partial S}{\partial T}\right)_{\rho, w}\left(\frac{\partial j_{0}}{\partial w}\right)_{T, \rho}-\left(\frac{\partial j_{0}}{\partial T}\right)_{\rho, w}^{2}\right)^{-1}>0
\end{aligned}
$$


corresponds to the usual requirement of the heat capacity positivity. It is shown below that $\left(\partial j_{0} / \partial w\right)_{T, \rho}>0$, hence the last inequality eventually becomes

$$
\left(\frac{\partial S}{\partial T}\right)_{\rho, w}\left(\frac{\partial j_{0}}{\partial w}\right)_{T, \rho}-\left(\frac{\partial j_{0}}{\partial T}\right)_{\rho, w}^{2}>0
$$

- Positivity of the next group of minors is easily verified with the following transformation

$$
\mathfrak{H}^{\prime}=\left\|\frac{\partial\left(T, \mathbf{v}_{\mathbf{n}}, \rho, \mathbf{v}_{\mathrm{s}}\right)}{\partial\left(S, \mathbf{j}, \rho, \mathbf{v}_{\mathrm{s}}\right)}\right\|=\left\|\frac{\partial\left(T, \mathbf{j}, \rho, \mathbf{v}_{\mathrm{s}}\right)}{\partial\left(S, \mathbf{j}, \rho, \mathbf{v}_{\mathrm{s}}\right)}\right\|\left\|\frac{\partial\left(T, \mathbf{v}_{\mathrm{n}}, \rho, \mathbf{v}_{\mathrm{s}}\right)}{\partial\left(T, \mathbf{j}, \rho, \mathbf{v}_{\mathrm{s}}\right)}\right\| .
$$

Positivity of the minors $M_{2}, M_{3}, M_{4}$ is guaranteed if all minors of the second term in (6) are positive

$$
\begin{aligned}
\left\|\left(\frac{\partial \mathbf{j}}{\partial \mathbf{v}_{\mathrm{n}}}\right)_{T, \rho, \mathbf{v}_{\mathrm{s}}}\right\|^{-1}=\left\|\left(\frac{\partial \mathbf{j}_{0}}{\partial \mathbf{w}}\right)_{T, \rho}\right\|^{-1}= \\
\|\left(\begin{array}{cccc}
\left.\partial j_{0} / \partial w\right)_{T, \rho} & 0 & 0 \\
0 & j_{0} / w & 0 \\
0 & 0 & j_{0} / w
\end{array} \|^{-1} .\right.
\end{aligned}
$$

Here we used (3) and chose the direction of the $\mathbf{w}$ vector as the first coordinate. Our collection is therefore augmented by two more inequalities

$$
\begin{gathered}
\mathbf{j}_{0} \mathbf{w} \geq 0 \\
\left(\frac{\partial j_{0}}{\partial w}\right)_{T, \rho}>0 .
\end{gathered}
$$

- The same transformation applied to the biggest minors yields:

$$
\mathfrak{H}=\mathfrak{H}^{\prime}\left\|\frac{\partial\left(T, \mathbf{v}_{\mathrm{n}}, \mu+v_{\mathrm{s}}^{2} / 2-\mathbf{v}_{\mathbf{s}} \mathbf{v}_{\mathrm{n}}, \mathbf{j}-\rho \mathbf{v}_{\mathrm{n}}\right)}{\partial\left(T, \mathbf{v}_{\mathrm{n}}, \quad \mathbf{v}_{\mathrm{s}}\right)}\right\|=\mathfrak{H}^{\prime} \mathfrak{H}^{\prime \prime}
$$

Again, the minors $M_{5}, M_{6}, M_{7}$, and $M_{8}$ are positive if all nontrivial principal minors of $\mathfrak{H}^{\prime \prime}$ are positive. We use the thermodynamic identity to relate the chemical potential $\mu$ and the conventional pressure $p$

$$
\mathrm{d} \mu=\frac{\mathrm{d} p}{\rho}-\frac{S}{\rho} \mathrm{d} T-\frac{\mathbf{j}_{0}}{\rho} \mathrm{d} \mathbf{w}
$$

This gives

$$
\left(\frac{\partial\left(\mu+v_{\mathrm{s}}^{2} / 2-\mathbf{v}_{\mathbf{s}} \mathbf{v}_{\mathrm{n}}\right)}{\partial \rho}\right)_{T, \mathbf{v}_{\mathbf{n}}, \mathbf{v}_{\mathbf{s}}}=\left(\frac{\partial \mu}{\partial \rho}\right)_{T, \mathbf{w}}=\frac{1}{\rho}\left(\frac{\partial p}{\partial \rho}\right)_{T, w} .
$$


The following is an explicit representation of $\mathfrak{H}^{\prime \prime}$ sub-matrix corresponding to a four-dimensional sub-space $\rho, v_{\mathrm{s}}^{x}, v_{\mathrm{s}}^{y}, v_{\mathrm{s}}^{z}$; as before we let the $x$-axis run along $\mathbf{w}$ direction. Using (3) we obtain

$$
\left\|\begin{array}{cccc}
(\partial p / \partial \rho)_{T, w} / \rho & \left(\partial j_{0} / \partial \rho\right)_{T, w}-w & 0 & 0 \\
\left(\partial j_{0} / \partial \rho\right)_{T, w}-w & \rho-\left(\partial j_{0} / \partial w\right)_{T, \rho} & 0 & 0 \\
0 & 0 & \rho-j_{0} / w & 0 \\
0 & 0 & 0 & \rho-j_{0} / w
\end{array}\right\| \text {. }
$$

Appropriate inequalities are:

$$
\left(\frac{\partial p}{\partial \rho}\right)_{T, w}>0
$$

which is literally a generalized (to a non-zero inter-component velocity $w$ ) positive compressibility requirement,

$$
\begin{gathered}
j_{0}<w \rho \\
\left(\frac{\partial p}{\partial \rho}\right)_{T, w}\left(\rho-\left(\frac{\partial j_{0}}{\partial w}\right)_{T, \rho}\right)-\rho\left(\left(\frac{\partial j_{0}}{\partial \rho}\right)_{T, w}-w\right)^{2}>0 .
\end{gathered}
$$

Inequalities (5), (7), (8), (9), (10), and (11) are sufficient conditions for the thermodynamic stability.

\section{DISCUSSION}

In a "stopped-normal-component" arrangement, the mass flux $\mathbf{f}$ with respect to the normal component may become more convenient than $\mathbf{j}_{0}$ - the mass flux relative to the superfluid one. The obvious relation between them $f=\rho w-j_{0}$ leads to the following reformulation of the inequalities:

$$
\begin{gathered}
\mathbf{f w}<0, \quad f<w \rho \\
0<\left(\frac{\partial f}{\partial w}\right)_{\rho, T}<\rho, \\
\left(\frac{\partial S}{\partial T}\right)_{\rho, w}\left(\rho-\left(\frac{\partial f}{\partial w}\right)_{T, \rho}\right)>\left(\frac{\partial f}{\partial T}\right)_{\rho, w}^{2}, \\
\left(\frac{\partial p}{\partial \rho}\right)_{T, w}\left(\frac{\partial f}{\partial w}\right)_{\rho, T}>\rho\left(\frac{\partial f}{\partial \rho}\right)_{w, T}^{2} .
\end{gathered}
$$


As a simple application of the derived inequalities, consider them at $w=0$. From (12), (13), (14), and (15) we get

$$
\begin{gathered}
\left(\frac{\partial S}{\partial T}\right)_{\rho, w}>0, \quad\left(\frac{\partial p}{\partial \rho}\right)_{T, w}>0, \\
\rho>\left(\frac{\partial j_{0}}{\partial w}\right)_{T, \rho}>0 .
\end{gathered}
$$

Using conventional notation, last inequality reads

$$
\rho_{\mathrm{s}}>0, \quad \rho_{\mathrm{n}}>0 .
$$

\section{PHONON-ROTON MODEL}

Here, we provide a practical example of the stability criteria for real superfluid ${ }^{4} \mathrm{He}$. To calculate derivatives involved in the inequalities one must use the microscopic approach. Simple and clear Landau phonon-roton model works pretty well over wide temperature and velocity ranges. We use this model to calculate the contribution of these quasiparticles to the "modified" free energy in the frame of reference of the superfluid component:

$$
\widetilde{\mathcal{F}}_{0}=\mathcal{F}_{0}-\mathbf{w} \mathbf{j}_{0}, \quad \mathrm{~d} \widetilde{\mathcal{F}}_{0}=-S \mathrm{~d} T-\mathbf{j}_{0} \mathrm{~d} \mathbf{w} .
$$

This potential is obtained from the excitation spectrum with the conventional formula

$$
\widetilde{\mathcal{F}}_{0}=T \int \ln \left(1-\exp \left(\frac{\mathbf{p w}-\epsilon(p)}{T}\right)\right) \frac{\mathrm{d} \mathbf{p}}{(2 \pi \hbar)^{3}} .
$$

We denoted the excitation energy $\epsilon(p)$, which is given for two branches by the expressions

$$
\epsilon_{\mathrm{ph}}(p)=c p, \quad \epsilon_{\mathrm{r}}(p)=\Delta+\left(p-p_{0}\right)^{2} /(2 m) .
$$

Subscripts distinguish the quantities related to phonons and rotons, $c$ is the sound velocity, $\Delta$ is the roton energy gap, $m$ is the effective mass, and $p_{0}$ is the momentum at the roton minimum (we take numerical data from Refs. [6], [7]: $\rho=0.145 \mathrm{~g} / \mathrm{cm}^{3}, \Delta=8.7 \mathrm{~K}, m=0.16 m_{\mathrm{He}}, p_{0} \rho^{-1 / 3} \hbar^{-1}=3.673 \cdot 10^{8} \mathrm{~g}^{-1 / 3}, c=$ $238 \mathrm{~m} / \mathrm{s}, \partial \Delta / \partial \rho=-0.47 \cdot 10^{-14} \mathrm{~cm}^{5} \mathrm{~s}^{-2}, \partial \mathrm{m} / \partial \rho=-0.45 \cdot 10^{-23} \mathrm{~cm}^{3}, \partial c / \partial \rho=$ $\left.467 \cdot 10^{3} \mathrm{~cm}^{4} \mathrm{~s}^{-1} \mathrm{~g}^{-1}\right)$. A small dimensionless parameter $m \Delta / p_{0}^{2} \sim 0.03 \ll 1$ ensures, e.g., that the Landau critical velocity is determined by $v_{\mathrm{L}}=\Delta / p_{0}$.

When integrated, these dispersion laws give the following contribution to the free energy:

$$
\widetilde{\mathcal{F}}_{0}=-\frac{T^{4} \pi^{2}}{90 \hbar^{3} c^{3}}\left(1-\frac{w^{2}}{c^{2}}\right)^{-2}-\frac{T^{5 / 2} m^{1 / 2}}{2^{1 / 2} \pi^{3 / 2} \hbar^{3}} \frac{p_{0}}{w} \sinh \frac{w p_{0}}{T} \exp \left(-\frac{\Delta}{T}\right) .
$$

One can obtain all thermodynamic variables by differentiating this potential. Namely

$$
S=-\left(\partial \widetilde{\mathcal{F}}_{0} / \partial T\right)_{w, \rho}, \quad j_{0}=-\left(\partial \widetilde{\mathcal{F}}_{0} / \partial w\right)_{T, \rho} .
$$




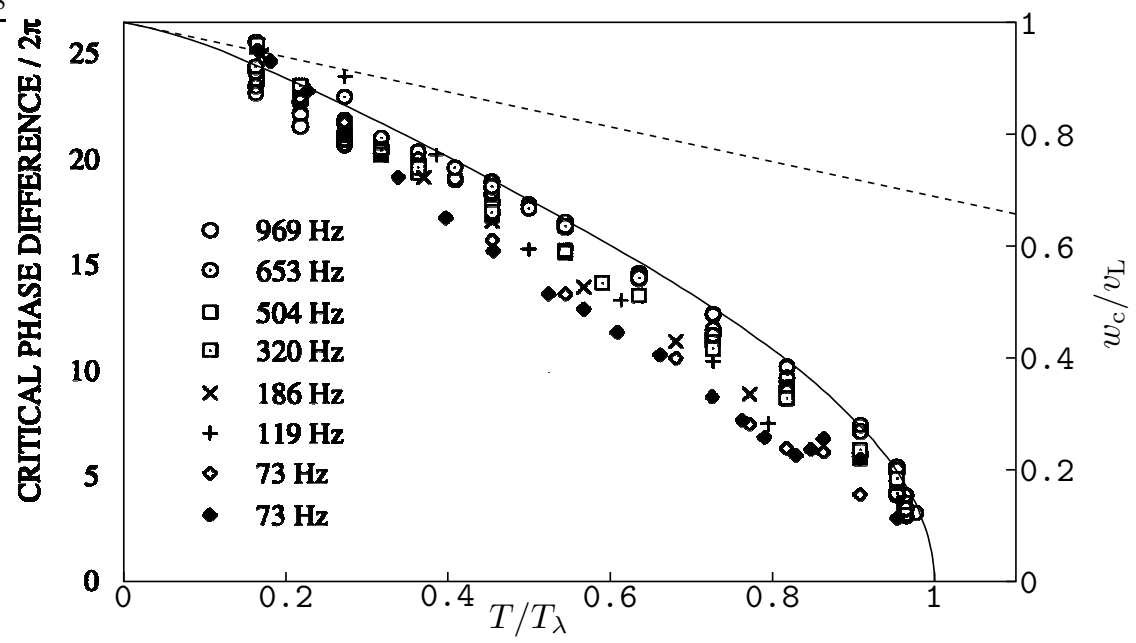

Figure 1: Critical velocity $w_{\mathrm{c}}$ versus temperature $T$. Dashed line corresponds to the equation $T=\Delta-p_{0} w$. Note that the condition $T<\Delta-p_{0} w$ holds true over entire stability domain. The "stability" critical velocity $w_{\mathrm{c}}$ coincides with the Landau critical velocity $v_{\mathrm{L}}$ at zero temperature and vanishes completely (see (16)) at the critical temperature $T_{\lambda}$. The curve is superimposed on the experimental graph of the critical phase difference over a $2 \mu \mathrm{m} \times 2 \mu \mathrm{m}$ aperture in a thin foil at various frequencies. 8 ]

We neglect the quasiparticle contribution to the pressure derivative.

At zero velocity the inequalities in Eq. (16) fail at the $\lambda$-point. When the velocity is higher, the inequality (11) is the first to become invalid. At zero temperature, the critical (the highest possible without stability violation) velocity tends to the Landau critical velocity $v_{\mathrm{L}}^{\dagger}$. Entire stability region is plotted in Fig. 1 and Fig. 2 The liquid is unstable above the curves.

In Fig. [1 the critical velocity is plotted with the solid line. Scaled experimental data 8 are presented in the same graph. High frequency points follow the shape of the theoretical curve, but at low frequencies sufficient discrepancy is observed. This might be explained by the long time required for vortex nucleation. At higher frequencies the thermodynamic stability limit is observed while lower frequency critical velocity is cut off by vortices. Whatever feasible explanation this might be, the following warning must be taken into account when experimental data on critical phase difference and our predictions are compared. Assumption that the critical velocity is determined by the stability limit implies that the hydrodynamic equations inside orifice are essentially nonlinear. Par-

\footnotetext{
${ }^{\dagger}$ Note that for systems where all quasiparticles can be described hydrodynamically (in other words, systems without roton branch) inequality (II) at zero temperature includes a condition $(\partial p / \partial \rho)_{T, w}-w^{2}>0$, i.e., $w<c$.
} 
ticularly, one can not consider the superfluid component as an incompressible fraction of the liquid. In other words the phase shift across the length of the orifice is not proportional to the maximum velocity attained in the orifice.

In Fig. 2 the critical energy flux is plotted. In typical arrangement the energy is carried by the superfluid counterflow, where normal and superfluid velocities are directed oppositely to each other to keep zero net mass flux. In zero mass flux frame the condition $j=0$ yields

$$
Q=T S\left(w-\frac{j_{0}}{\rho}\right)+\left(w-\frac{j_{0}}{\rho}\right)^{2} j_{0}
$$

for the energy flux.

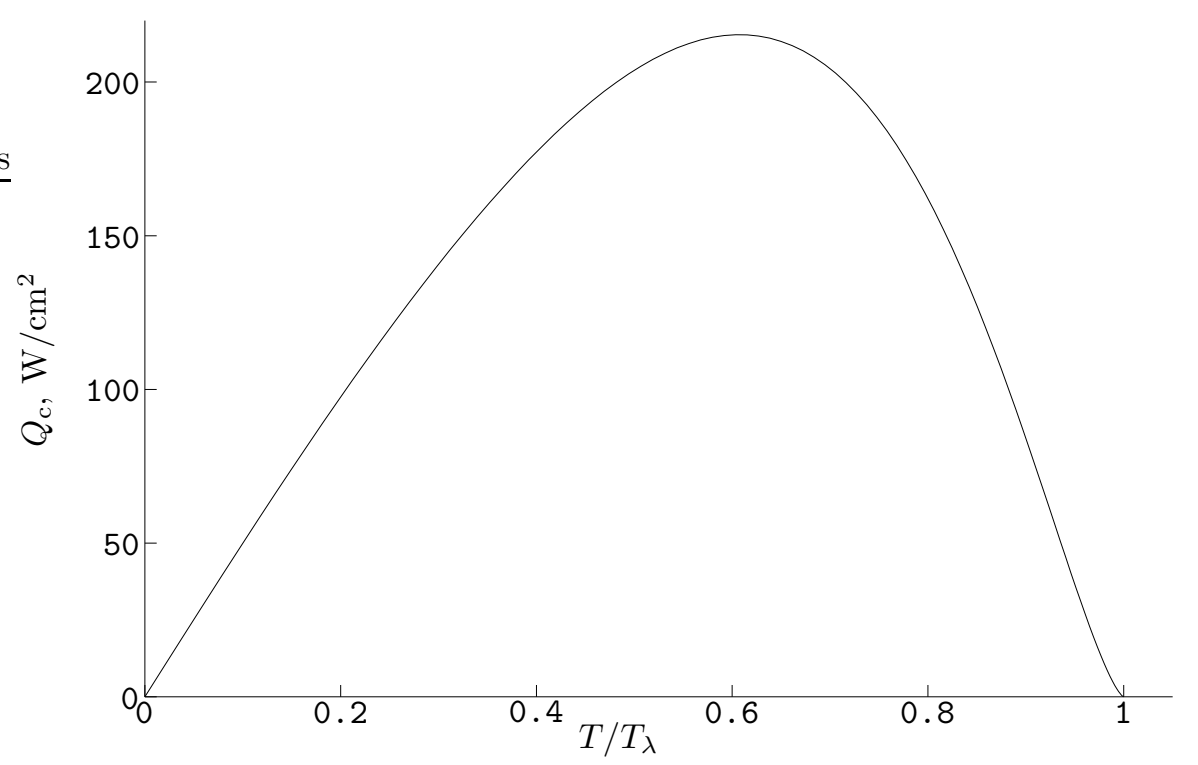

Figure 2: Critical energy flux (at zero mass flux) $Q_{c}$ versus temperature $T$.

\section{CRITICAL BEHAVIOR}

To find the behavior of the critical velocity near the $\lambda$-point, scaling arguments should be used. We assume that the low velocity expansion of the irregular part of thermodynamic potential near $T_{\lambda}$ starts with

$$
A \tau^{2-\alpha}+\rho_{s} \frac{v_{\mathrm{s}}^{2}}{2}-B \tau^{x} v_{\mathrm{s}}^{4}
$$

where $A$ and $B$ are constants, $\tau=\left(T_{\lambda}-T\right) / T_{\lambda}, \alpha$ is the critical index of the specific heat $C_{p} \propto \tau^{-\alpha}$, the "superfluid density" $\rho_{s}$ follows Josephson scaling relation [9] $\rho_{s} \propto \tau^{(2-\alpha) / 3}$, and the index $x$ will be determined below. According 
to the hypothesis of scale invariance, all terms in (18) are of the same order in the fluctuation region. This yields $\tau^{x} \propto \rho_{s}^{2} \tau^{\alpha-2}$ and $x=(\alpha-2) / 3$. On the other hand, the stability is destroyed by the velocity at which the linear approximation fails, i.e.,

$$
w_{c} \propto \sqrt{\rho_{s} / \tau^{x}} \propto \tau^{(2-\alpha) / 3} .
$$

Current value for the specific heat index[10] $\alpha=-0.0127 \pm 0.0003$ gives 0.671 exponent for the critical velocity

Extensive data near the $\lambda$-point are available in the critical energy flux experiments. 2] In the vicinity of the transition, Eq. (17) is reduced to $Q \approx T S\left(w-j_{0} / \rho\right)$ and the critical flux is determined by

$$
Q_{c} \propto \rho_{s} w_{c} \propto \tau^{(4-2 \alpha) / 3} \approx \tau^{1.342},
$$

which is in moderate agreement with experimental value of the exponent $1.23 \pm$ 0.02 .

\section{CONCLUSION}

Experimentally, the superfluidity breakdown is believed to have the following nature. 1] Until the aperture size is too small, the critical velocity does not depend on the temperature and increases as the size decreases. This is the very behavior that is specific to the Feynman vortex-related critical velocity.

When the orifice width is narrow enough, or the temperature is close enough to $T_{\lambda}$ the breakdown scenario and its features change. The critical velocity does not depend on the aperture any more but decreases when the temperature increases. This behavior is commonly associated [1] with the Iordanski-LangerFisher mechanism. 11] Nevertheless, this association lacks numerical comparison because no reliable information about the actual orifice shape is available.

On the other hand experimentally observed behavior of the critical velocity can be attributed to the suggested stability criterion. In other words, we provide an alternative explanation of experimental results based on an assumption that in narrow orifices the thermodynamic limit of $w_{\mathrm{c}}$ is reached.

We should also note that our approach to the critical velocity as a stability limit is similar to that used by Kramer. 12. Actually, the inequality he employed is not a thermodynamic one, but numerical results for the critical velocity he obtained using the phonon-roton model do not deviate much from those plotted in Fig. 1]

\section{ACKNOWLEDGMENTS}

Opportunity to write the paper for the JLTP issue dedicated to the memory of Olli Lounasmaa, the person contributed so much to the physics of superfluidity and to the field of low temperatures in general, is a great honor to us. It is also 
very important for us that Olli was one of the founders of the close scientific collaboration between Finland and Russia which turned out to be so important for the world science.

It is a pleasure for us to thank I.A. Fomin for useful discussions. One of us (LM) would also like to thank University of New Mexico for its hospitality during writing this paper. This work was supported in parts by INTAS grant 01-686, CRDF grant RP1-2411-MO-02, RFBR grant 03-02-16401, and RF president program.

\section{References}

[1] E. Varoquaux, W. Zimmermann, and O. Avnel, in Excitations in TwoDimensional and Three-Dimensional Quantum Fluids, edited by A.F.G. Wyatt and H.J. Lauter (NATO ASI Series, Plenum Press, New YorkLondon, 1991), p. 343.

[2] R.V. Duncan, G. Ahlers, and V. Steinberg, Phys. Rev. Lett. 601522 (1988).

[3] A.F. Andreev, L.A. Melnikovsky, JETP Lett. 78574 (2003).

[4] L.D. Landau, E.M. Lifshitz, Statistical Physics, part 1 (Pergamon Press, Oxford, 1980).

[5] I.M. Khalatnikov, An Introduction to the Theory of Superfluidity. (W.A. Benjamin, New York-Amsterdam 1965).

[6] R.J. Donnelly, P.H. Roberts, JLTP 27, 687 (1977).

[7] R.J. Donnelly, J.A. Donnelly, R.N. Hills, JLTP 44, 471 (1981).

[8] J.A. Flaten, C.A. Lindesmith, W. Zimmermann, JLTP 101, 743 (1995).

[9] B.D. Josephson, Phys. Lett. 21, 608 (1966)

[10] J.A. Lipa, J.A. Nissen, D.A. Stricker, D.R. Swanson, T.C.P. Chui, cond-mat/0310163.

[11] J.S. Langer, J.D. Reppy, Prog. Low. Temp. Phys., vol. VI, ed. C.J.Gorter (North-Holland, Amsterdam, 1970) ch.1.

[12] L. Kramer, Phys. Rev. 179, 149 (1969). 